\title{
Spatial distribution control of polymer nanoparticles by liquid crystal disclinations
}

\author{
Kenji Higashiguchi ${ }^{1}$, Kei Yasui $^{2}$, Masaaki Ozawa ${ }^{2}$, Keisuke Odoi ${ }^{2}$ and Hirotsugu Kikuchi ${ }^{3}$ \\ A perylene-labeled hyper-branched polymer with a mesogenic shell was observed to migrate toward a field with a large distortion \\ of directors. This polymer was captured by disclination lines of $1 / 2$ strength in its nematic and chiral nematic phases using a \\ confocal fluorescence microscope. In particular, the well-aligned particle array of the hyper-branched polymers was produced \\ by the chiral nematic phase confined in a wedge cell. The hyper-branched polymer with its mesogenic shell was completely \\ dissolved into an isotropic phase. Immediately after a phase transition to the chiral nematic phase, numerous droplets appeared \\ over a wide area owing to phase separation, and the free droplets moved toward the disclination lines via Brownian motion. \\ Finally, the droplets were rapidly attracted to the disclination lines and trapped within them.
}

Polymer Journal (2012) 44, 632-638; doi:10.1038/pj.2012.44; published online 11 April 2012

Keywords: confocal fluorescence polarizing microscope; disclination line; fluorescence; hyper-branched polymer; liquid crystal

\section{INTRODUCTION}

Controlling the spatial arrangements of molecules or particles on different length scales is one of the crucial issues of bottom-up nanotechnology. ${ }^{1,2}$ Recently, dispersions of colloidal particles and polymers in nematic or cholesteric phases have attracted considerable attention because they demonstrate a diversity of self-assembled arrangements, such as linear or circle chains and $2 \mathrm{D}$ lattices. ${ }^{3-6}$ The long-range force of self-assembly originates from the elastic energy of the distortions produced around the particles in the nematic phase. $^{7-14}$ The particles have also been observed to be attracted to and to migrate toward a disclination or domain boundary in the nematic field. Because the director distortions of nematic liquid crystals $^{15,16}$ are large near the disclination, the elastic energy is higher near the disclination than in uniform director regions. ${ }^{3}$ The particles near the disclination might be exposed to an attractive force toward the disclination core, where the highest director gradient is formed.

In terms of particle polymers, hyper-branched polymers (HBPs), a novel class of polymer with highly branched structures and large numbers of end groups, are attractive because dendritic chain structures can be produced via an one-pot reaction, and the chemical structure and molecular weight of HBPs can be easily modified. ${ }^{17-23}$ Therefore, the physical properties of the HBPs, such as solubility to a host phase, molecular weight, viscosity, refractive index and fluorescence, can be easily controlled. If the spatial distribution and arrangement of HPBs are further controlled, the range of applications of these materials will expand extensively, especially in photonics and electronics.

In this paper, we synthesize perylene-labeled HBPs with mesogenic shells (1) that can be dispersed in a nematic liquid crystal and study the behavior of the HBPs captured by disclinations of the nematic phase. A well-aligned periodic array of HBP-droplet chains is successfully built subsequently using a topologically induced disclination array in a chiral nematic phase.

\section{EXPERIMENTAL PROCEDURE}

Synthesis

A methacrylate monomer with initiator 2-(N,N-diethyldithiocarbamyl)ethyl methacrylate ${ }^{17}$ was synthesized via the reaction of 2-chloroethyl methacrylate with $N, N$-diethyldithiocarbamate sodium salt in acetone. The details concerning the synthesis and purification have been provided elsewhere. ${ }^{17}$

A liquid crystalline monomer, 5-(4-cyanobiphenyl-4'-yloxy)pentyl methacrylate (3) was prepared from 4-cyano- $4^{\prime}$-hydroxybiphenyl using a procedure described by Craig et al. ${ }^{24}$

The precursor, $N$-(1-hexylheptyl)perylene-3,4:9,10-tetracarboxy-3,4-anhydride-9, 10 -imide, ${ }^{25}$ was prepared according to a procedure similar to that found in the literature. ${ }^{26}$ The synthesis of methacryl derivatives is illustrated in Supplementary Information Scheme S1.

\begin{abstract}
Synthesis of the core polymer
Photo-copolymerizations in tetrahydrofuran $(109.7 \mathrm{~g})$ solution of $2-(\mathrm{N}, \mathrm{N}-$ diethyldithiocarbamyl)ethyl methacrylate $(12.2 \mathrm{~g})$ with perylene methacrylate derivative $2(60.9 \mathrm{mg}$, feed fraction of $2=0.5 \mathrm{wt} \%$, monomer concentration $=10 \mathrm{wt} \%$ ) were performed via irradiation with ultraviolet (UV) light in a sealed glass ampoule under $\mathrm{Ar}$ atmosphere at room temperature for $7 \mathrm{~h}$. After the photoreaction, the copolymer was separated by preparative gel permeation chromatography (GPC) because perylene derivative 2 was hardly soluble in methanol, which was the reprecipitation solvent. The isolated HBP in tetrahydrofuran was recovered by precipitation in
\end{abstract}

${ }^{1}$ Department of Synthetic Chemistry and Biological Chemistry, Graduate School of Engineering, Kyoto University, Kyoto, Japan; ${ }^{2}$ Nissan Chemical Industries, Funabashi, Japan and ${ }^{3}$ Institute for Material Chemistry and Engineering, Kyushu University, Kasuga, Japan

Correspondence: Professor H Kikuchi, Institute for Material Chemistry and Engineering, Kyushu University Kasuga Park 6-1, Kasuga 816-8580, Japan.

E-mail: kikuchi@cm.kyushu-u.ac.jp

Received 28 December 2011; revised 2 February 2012; accepted 6 February 2012; published online 11 April 2012 
methanol at $-10{ }^{\circ} \mathrm{C}$ and precipitated as a pink powder. GPC analysis produced a conversion of $69 \%$ and only $1.0 \mathrm{~g}$ of the HBP was isolated. The polystyrene-reduced $M_{w}$ and $M_{w} / M_{n}$ of the HBP with perylene were measured to be $6.96 \times 10^{4}$ and 5.64 , respectively.

\section{Synthesis of the grafted polymer 1}

A tetrahydrofuran $(50 \mathrm{ml})$ solution of the recovered perylene-labeled HBP $(265 \mathrm{mg}, 1.0 \mathrm{mmol}), N, N, N^{\prime}, N^{\prime}$-tetraethylthiuram disulfide $(297 \mathrm{mg}$, $1.0 \mathrm{mmol})$, liquid-crystalline monomer $3(1.09 \mathrm{~g}, 3.0 \mathrm{mmol})$, and dodecyl methacrylate $(763 \mathrm{mg}, 3.0 \mathrm{mmol})$ in a sealed glass ampoule under Ar atmosphere was exposed to UV irradiation for $2 \mathrm{~h}$. After the photoreaction, the grafted polymer was recovered by precipitation in methanol at $-10{ }^{\circ} \mathrm{C}$, and a perylene-labeled HBP with a liquid-crystalline shell 1 was precipitated as a pink powder $(250 \mathrm{mg})$. The molar ratio of 2-(N,N-diethyldithiocarbamyl)ethyl methacrylate and mesogenic methacrylate 3 was measured by ${ }^{1} \mathrm{H}$ NMR as 97.8 and 2.1, respectively. The ratio of the core to shell was expected to be 95.8:4.2. The polystyrene-reduced $M_{w}$ and $M_{w} / M_{n}$ of the HBP with perylene were measured as $7.13 \times 10^{4}$ and 6.87 , respectively.

\section{Materials}

GPC and GPC-MALS analyses were performed to estimate the conversion factor between the absolute and polystyrene-reduced molecular weight for HBP. The core-HBP from the polymerization of 2-( $N, N$-diethyldithiocarbamyl)ethyl methacrylate without perylene 2 was analyzed as follows: GPC $M_{n}=1.55 \times 10^{4}, \quad M_{w}=5.15 \times 10^{4}$, and $M_{w} / M_{n}=3.33$; GPC-MALS $M_{n, \mathrm{MALS}}=3.79 \times 10^{4}, M_{w, \mathrm{MALS}}=1.08 \times 10^{5}$, and $M_{w, \mathrm{MALS}} / M_{n, \mathrm{MALS}}=2.84$.

The liquid crystal materials used in this study consisted of nematic 4-cyano-4'-pentylbiphenyl (5CB). A chiral dopant, 2,5-bis-[4'-(hexyloxy)phenyl-4-carbonyl] -1,4;3,6-dianhydride-D-sorbitol (ISO-(6OBA) $)_{2}$ ), was used to induce a chiral nematic phase (ca. $1-2 \mathrm{wt} \%)$. The perylene-labeled HBP with LC shell was added to isotropic LC, and various sandwiched glass cells were filled with the homogeneous mixture. To obtain a nematic LC in a thick cell, a glass cell with $75 \mu \mathrm{m}$ spacers was filled with a homogeneous mixture at $50{ }^{\circ} \mathrm{C}$ and quenched to room temperature. The initial in-plane defects were instantly fixed upon exposure to UV irradiation $(365 \mathrm{~nm}$, L2859-01, Hamamatsu Photonics, Hamamatsu, Japan) for several minutes. However, for the chiral nematic LC in Grandjean-Cano wedge cells $(\tan \theta=0.0078)$, photopolymerization was not required because the defects formed in the equilibrium state.

Imaging experiments were conducted with a transmission optical microscope (OPTIPHOT2-POL, Nikon, Tokyo, Japan) with a $\times 10$ objective lens (NA (numerical aperture) $=0.21$, Nikon) and a confocal fluorescence polarizing microscope (LSM 510, Carl Zeiss, Tokyo, Japan). The excitation beam $(488 \mathrm{~nm}$, Ar laser) was split using a beam splitter (HFT488) and was focused by an objective into a small volume within the LC slab. The fluorescent light from this volume was detected by a photomultiplier tube in the spectral region from $535-590 \mathrm{~nm}$. The pinhole size was adjusted according to the magnification and NA of the objective, which was $51 \mu \mathrm{m}$ and $92 \mu \mathrm{m}$ for the $\times 10(\mathrm{NA}=0.45$, Plan-Apochromat, Carl Zeiss $)$ and $\times 20(\mathrm{NA}=0.5$, PlanNeofluar, Carl Zeiss) objective lenses, respectively. Because the excitation beam was polarized, the anisotropy of the nematic LC could be observed. Small HBP concentrations and the light intensities were needed to avoid director field distortions resulting from the Janossy effect, that is, the light-induced reorientation of small-sized fluorescent HBP. ${ }^{27}$

\section{RESULTS AND DISCUSSION}

\section{Materials}

We synthesized perylene-labeled ${ }^{28-32}$ HBPs with mesogenic shells ${ }^{24-26}$ (1) as shown in Scheme 1 that could be dispersed in a nematic liquid crystal and studied the behavior of HBPs captured by disclinations using confocal fluorescence polarizing microscopy. ${ }^{33,34}$ A perylene derivative with a methacryl group (2) was introduced to the HBP using photo-copolymerization. Additional graft polymerization of a liquid-crystalline methacryl monomer, 5-(4-cyanobiphenyl-4'yloxy)pentyl methacrylate (3), and dodecyl methacrylate mixture was performed after synthesizing the HBP, resulting in the formation of a mesogenic shell surrounding the HBP to improve its solubility in 5CB. The chemical structures are presented in the Supplementary Information section. A well-aligned periodic array of HBP-droplet chains was successfully built using a topologically induced disclination array in a chiral nematic phase. We used 4-cyano- 4 -pentylbiphenyl $(5 \mathrm{CB})$ as the nematic LC. To induce the creation of a chiral nematic phase, the chiral dopant, 2,5-bis-[4'-(hexyloxy)-phenyl-4-carbonyl]-1,4;3,6-dianhydrideD-sorbitol (ISO-(6OBA) 2 ), was doped in nematic 5CB.

A dithiocarbamate with a primary aliphatic alkyl bond, such as methacrylate, has little tendency to be homolytically cleaved in comparison with a conjugated alkyl, such as benzyl. In general, the partially-branched polymers were obtained from the polymerization of 2-(N,N-diethyldithiocarbamyl)ethyl methacrylate, and therefore the viscosity ratio $g^{\prime}=\left[\eta_{\text {branched }}\right] /\left[\eta_{\text {linear }}\right]$ of methacrylate-HBP $\left(g^{\prime}=0.38-0.44\right)^{17}$ was larger than the value of benzyl-HBP $\left(g^{\prime}=0.16-0.28\right) .^{35}$

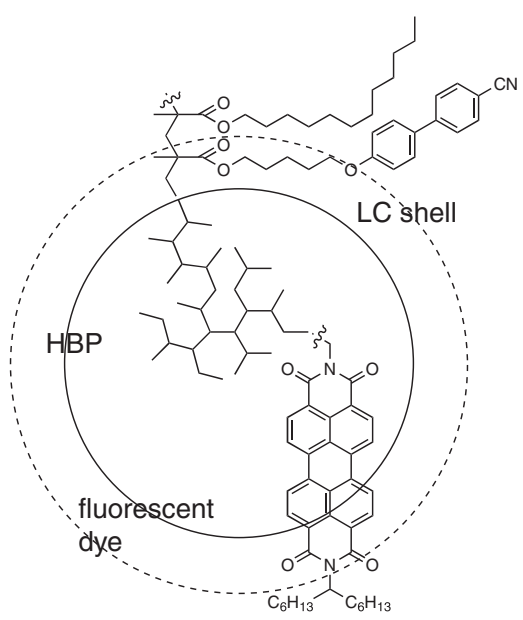

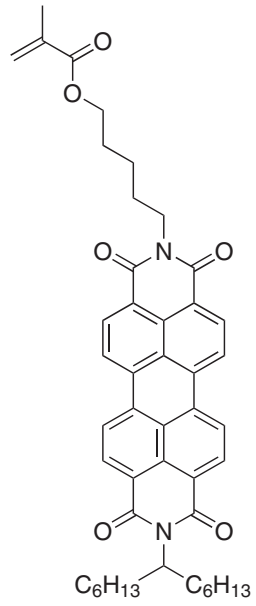

2<smiles>C=C(C)C(=O)OCCCCCOc1ccc(-c2ccc(C#N)cc2)cc1</smiles>

Scheme 1 Polymer $\mathbf{1}$, compounds 2 and $\mathbf{3}$. 
The solubility of HBPs was strongly dependent on the chemical structure of the shell surrounding the HBPs. The HBP without a shell and the HBP with a mesogenic shell prepared from only monomer 3 were insoluble in $5 \mathrm{CB}$ for both the nematic and isotropic phases. However, the HBP with the mesogenic shell prepared using $50 / 50 \mathrm{~mol} \%$ of 3 and dodecyl methacrylate exhibited good and poor solubility in $5 \mathrm{CB}$ for the isotropic and nematic phase, respectively. When 0.02 wt $\%$ of 1 was dissolved in $5 \mathrm{CB}$, the solution was completely homogeneous and transparent in the isotopic phase, whereas 1 precipitated in the nematic phase.

\section{Microscopic measurement of HBP 1 in the nematic phase}

The phase-separated behavior of 1 in $5 \mathrm{CB}$ was observed using a confocal fluorescence polarizing microscope ${ }^{36-42}$ which allows highspatial resolution of localized fluorescence-labeled molecules in a thick medium. ${ }^{43-45}$ When the solution of $5 \mathrm{CB}$ and $\mathbf{1}$ was in the isotropic phase at $50^{\circ} \mathrm{C}$, the observed field was uniformly and slightly bright using fluorescent light from 1 with a wavelength of approximately $535 \mathrm{~nm}$ over the entire specimen; that is, 1 was uniformly dispersed at a nearly molecular level in the isotropic phase of $5 \mathrm{CB}$. When the solution transformed into the nematic phase after quenching to ambient temperature, numerous small fluorescent droplets suddenly appeared during the phase transition. Because of the thick cell and lack of surface treatment of the substrate surface, many thread-like line defects occurred in the specimen in the nematic phase. The defects were unsteady and moved drastically just after the phase transition occurred. A number of disclinations merged with each other and subsequently disappeared. Finally, a Schlieren texture was obtained in which the line defects, the disclinations with strengths $s$ of $1 / 2$ or $-1 / 2$, were formed vertically with respect to the substrate surface. We observed that the fluorescent small droplets were trapped by the thread-like line defects, and the particles aggregated along these lines before the lines disappeared. To immobilize the line defects filled with 1, the specimen was irradiated with UV light, which induces chemical interconnection among 1 molecules. The HBPs underwent photo- and thermal-living radical polymerization that originated from the cleavage and recombination of the thiocarbonyl groups on the HBP surface. ${ }^{17-23}$ The living radical polymerization produced not only the additional chain polymerization of the monomers to the surface of the HBP, such as in graft polymerization, but also induced the addition of polymeric segments to the other polymeric segments at the surface of the HBP. Therefore, the HBPs in the disclination lines could be further polymerized using UV irradiation to immobilize these disclination lines before the mobilization or merging of these disclination lines and the formation of micrometer-sized HBP particles. Figures $1 \mathrm{a}$ and $\mathrm{b}$ present the polarizing optical micrograph and the confocal fluorescence polarizing micrograph, respectively, of a line defect after immobilization in the nematic phase. The thread-like line includes a core with two black brushes under crossed polarizers as shown in Figure 1a, indicating that the line is a disclination of $s=1 / 2$ or $-1 / 2$. Furthermore, the line was identified to be $-1 / 2$ because when the sample was rotated with respect to the axes of polarizers, the black brushes rotated in the same direction as the sample rotation. Figure $1 \mathrm{~b}$ clearly illustrates that the fluorescent small particles, the bright regions in Figure $1 \mathrm{~b}$, are captured and aggregated along the disclination line.

Microscopic measurement of HBP 1 in the chiral nematic phase Disclinations are formed in an equilibrium state when a chiral nematic phase is confined within a finite volume. When a Grandjean-Cano wedge-shaped cell is filled with chiral nematic

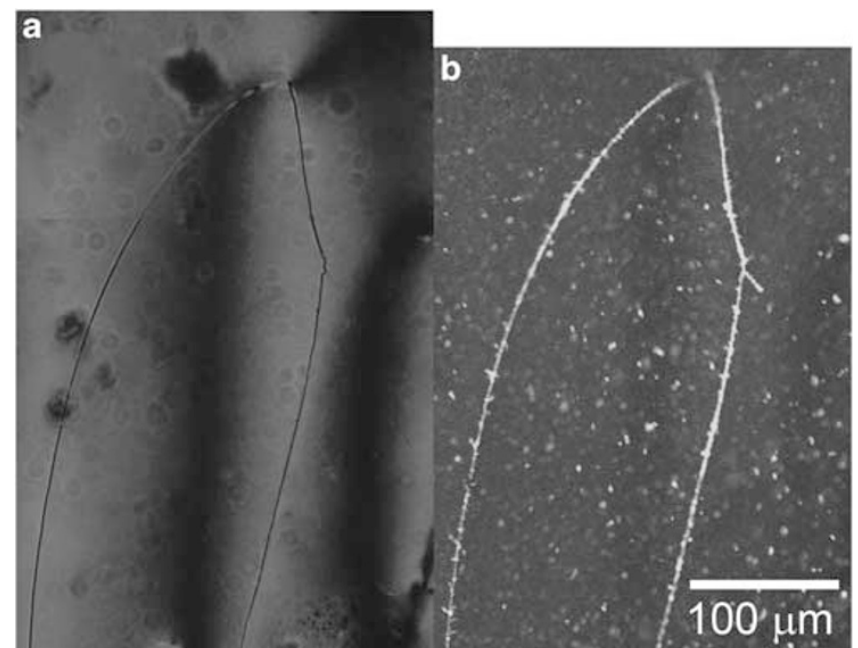

Figure 1 (a) Polarizing optical microscope and (b) confocal fluorescence polarizing microscope texture of a nematic liquid crystal with planar defects in a thick glass cell ( $75 \mu \mathrm{m}$ spacer). Bright spots imply the presence of the perylene-labeled HBP and the bright lines of (b) correspond to the disclination lines of (a); the disclination was defined as $s=-1 / 2$ (see Supplementary Information).

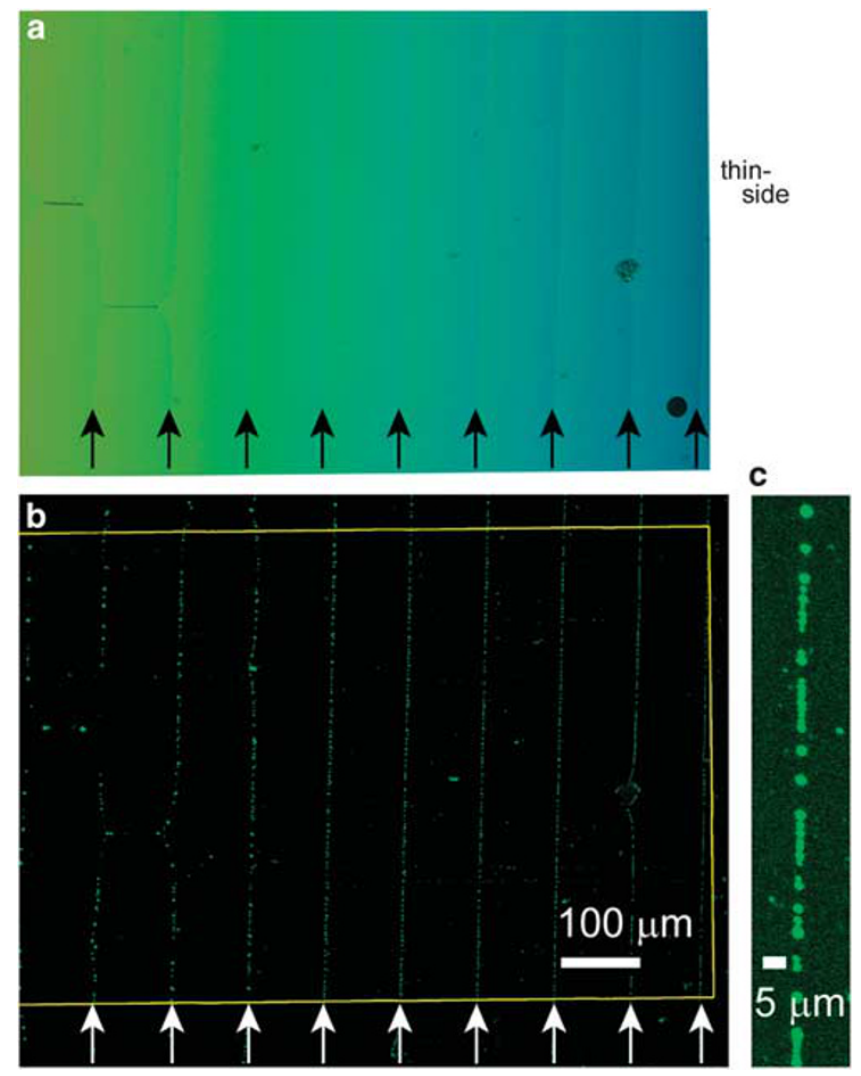

Figure 2 (a) Polarizing optical microscope and (b) confocal fluorescence polarizing microscope texture of periodically aligned defects in a GrandjeanCano wedge cell. The bright lines of (b) correspond to the disclination lines of (a): edge dislocations with Burgers vectors $b=p / 2$. The pitch was calculated to be $p=1.51 \mu \mathrm{m}$. (c) The magnified images near the disclination line of $(\mathbf{b})$. 
phase, regularly aligned defect lines of $|s|=1 / 2$ form in the cell perpendicular to the gradient of the cell thickness. The defect lines in the thin region of the cell are edge dislocations composed of a pair of $\tau$ and $\lambda$ disclinations. ${ }^{34,46}$ A wedge cell was filled with a mixture of $5 \mathrm{CB} / \mathrm{ISO}-(6 \mathrm{OBA})_{2} / \mathbf{1}=99.0 / 0.95 / \mathrm{ca} \cdot 0.020 \mathrm{wt} \%$ and observed using polarizing optical microscopy and confocal fluorescence polarizing microscopy. Figures $2 \mathrm{a}$ and $\mathrm{b}$ present photographs of an identical field in the cell observed using a polarizing optical microscope and a confocal fluorescence polarizing microscope, respectively. An array of periodically aligned defect lines was formed in the wedge cell, as illustrated in Figure 2a. It should be noted that the fluorescent particles consisting of $\mathbf{1}$ assembled along the defect lines, as shown in Figure $2 b$. From the magnified photograph presented in Figure $2 c$, the particles are approximately $1-2 \mu \mathrm{m}$ in diameter and are connected with one another in a line like a string of beads. Interestingly, the diameter of the particles increased as the cell thickness increased in the wedge cell.

Figure 3 presents snapshots at transitional periods of the phase transition from an isotropic phase to a chiral nematic phase in the wedge cell. For the isotropic phase (a), no particles were observed. Directly after the phase transition to the chiral nematic phase by quenching to ambient temperature, numerous particles appeared across a broad area (b) and were rapidly swept away by moving defect lines from the left (thick) to right (thin) side (c). After several hundred seconds, the defect lines almost stabilized; however, the free particles that were not trapped by the defect lines moved around via Brownian motion and sometimes coalesced with one another. Interestingly, the motion of the particles was not random; instead, in general, they migrated toward the left (thick) side and subsequently accumulated at the defect lines (d)-(l). Once the particles were trapped in the defect lines, their motion was limited to one dimension along the defect line. Thus, the disclinations are clearly shown to exert a long-range attractive force on the HBP particles. The time

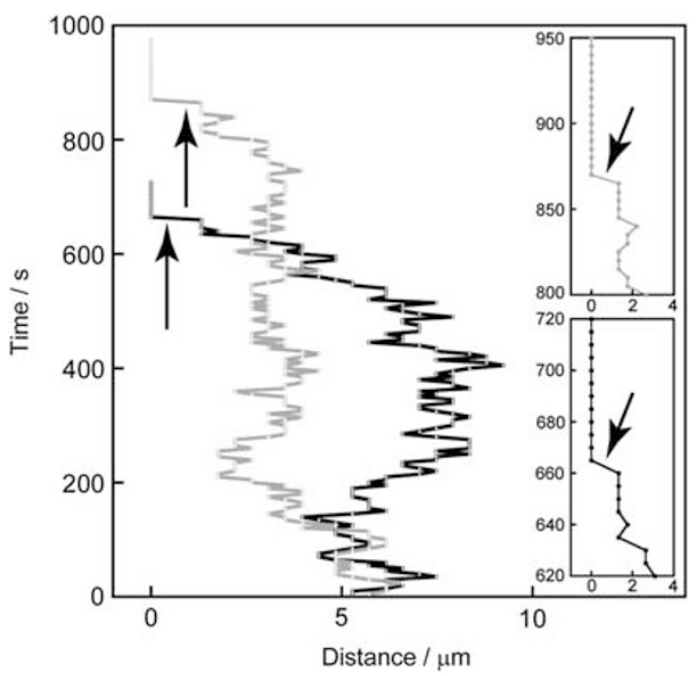

Figure 4 Time trace of the Brownian motion and migration of two droplets to a disclination line in the chiral nematic LC fluid. The black and gray solid lines indicate the particles $\beta$ and $\gamma$ in Figure 3, respectively. Motion along the $z$ axis was not measured. The arrows indicate migrations, and the insets illustrate the change of distance near the final migration.
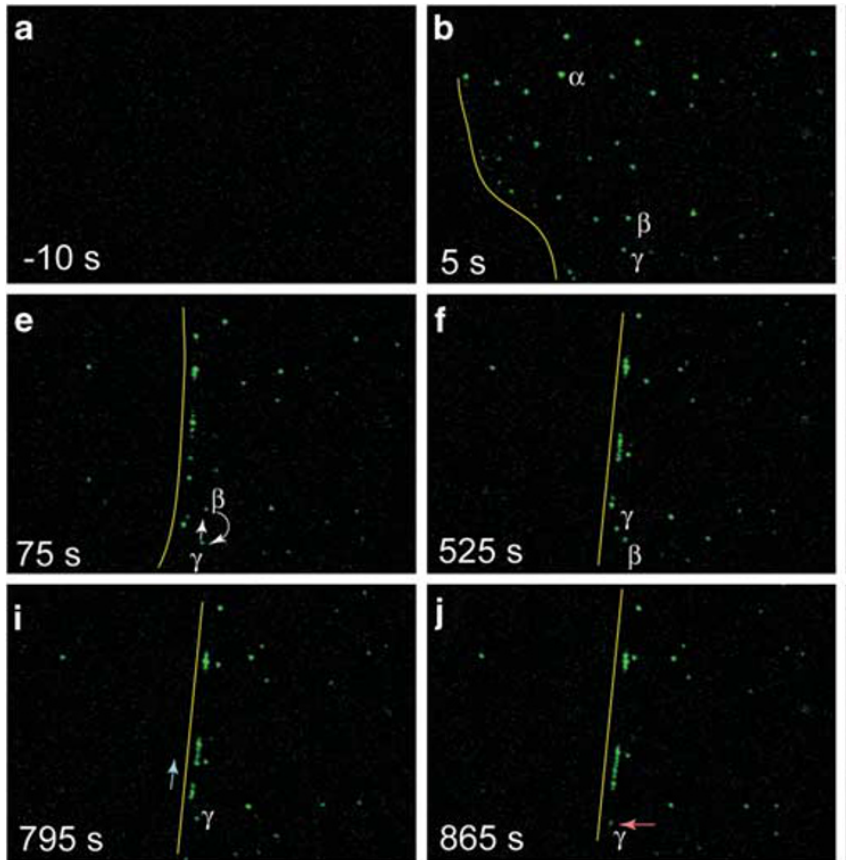
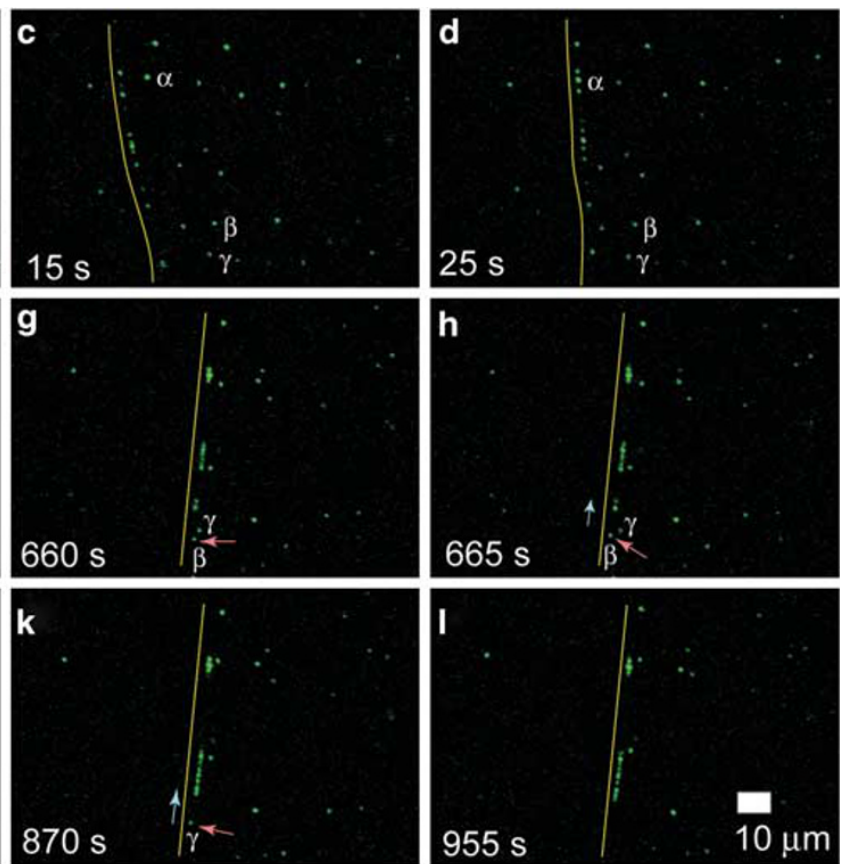

Figure 3 Particle dynamics in a chiral nematic liquid crystal near the disclination line. The micrographs were obtained using a confocal fluorescence polarizing microscope ( $5 \mathrm{~s}$ per frame). The time of the phase transition from the isotropic to chiral nematic phase was set as $0 \mathrm{~s}$. The yellow lines indicate the disclination lines (which are offset to the left for clarity), the white arrows indicate Brownian motion without migration, the pink arrows indicate the migration, and the sky-blue arrows indicate one-dimensional movement along the disclination line. (a) In the isotropic phase, no particles were observed, $(\mathbf{b}, \mathbf{c})$ many particles appeared after quenching and the disclination line moves to right (thin side) captured particles (e.g., $\alpha$ ), (d-f) Brownian movement (e.g., $\beta$ and $\gamma$ ) was observed (see Supplementary Information), (g-i) migration to the disclination line ( $\beta$ ) and one-dimensional motion were observed, and (j-I) the other droplet $\gamma$ also migrated. 


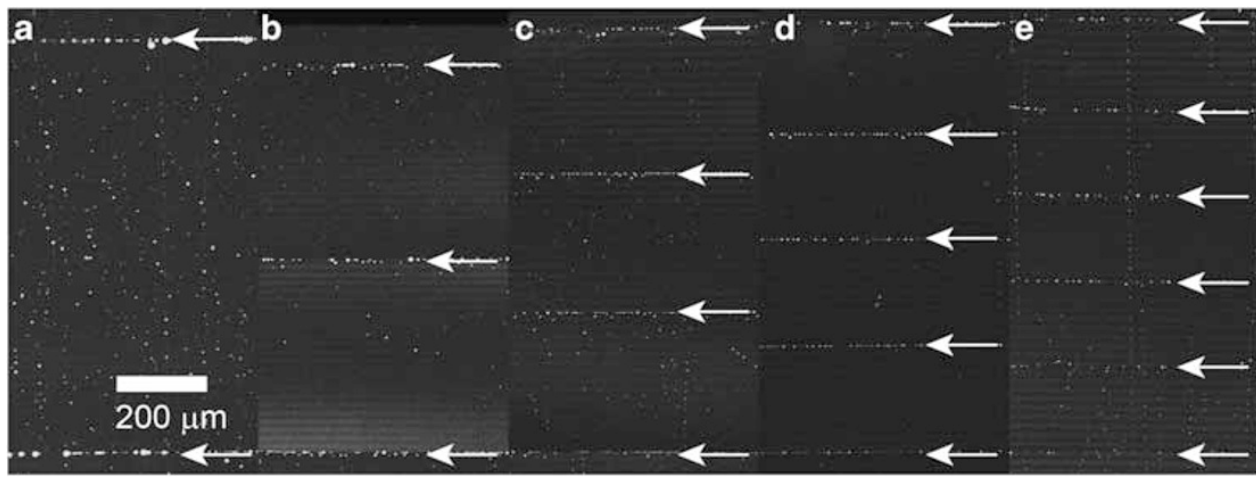

Figure 5 The fluorescence microscopic observations of the HBP alignments in the wedge cell with chiral dopant concentrations of (a) 0.126 wt\% (b) $0.252 \mathrm{wt} \%$ (c) $0.379 \mathrm{wt} \%$ (d) $0.497 \mathrm{wt} \%$ and (e) $0.616 \mathrm{wt} \%$. The HTP value was calculated as $55.2 \pm 2.0 \mu \mathrm{m}^{-1}$.

dependence of the distances between a droplet and the disclination line at which the droplet was finally trapped is illustrated in Figure 4. The droplets moved randomly in regions far from the disclination; however, in general, most of the droplets drifted from the thin regions to the thick regions. As a result, the droplets gradually approached the disclination line. During the final stage of migration into the disclination, the droplets were rapidly attracted to the disclination line and traveled approximately $1.3 \mu \mathrm{m}$ to reach it. After that movement, the Brownian motion was restricted to 1 dimension along the disclination line. However, 3-dimensional Brownian motion could not be measured using a confocal fluorescence polarizing microscope because of the wide range of observations and the rapid scan. Detailed studies of similar behavior have been reported elsewhere. ${ }^{7-14}$

The spacing of a disclination array of regular intervals can be determined by the pitch length of the chiral nematic phase, which is proportional to the reciprocal of the concentration of the chiral dopant and the angle between the two substrates of the wedge cell. We regulated the spacing of the disclination lines by changing the concentration of the chiral dopant. We can regulate the spacing of the HBP chain array using the disclination array, which can serve as a template for the HBP arrangement. Fluorescence micrographs of wellaligned chains of HBP droplets with various spacings are presented in Figure 5.

\section{Molecular weight dependence of the solubility of HBP 1}

The unfractionalized HBPs were used in the study described above. However, the molecular weight of the HBPs should influence the phase separation in a liquid crystal because the miscibility of a polymer with liquid crystals depends strongly on the molecular weight of the polymer. The perylene-labeled HBP 1 was fractionalized by GPC, as described in Table 1, to examine the molecular weight dependence of the phase separation. For HBP $1 \mathrm{a}\left(M_{n} \sim 4.4 \times 10^{3}\right)$ and HBPs with a higher molecular weight, phase separation occurred in both the nematic and chiral nematic phases, similar to that in unfractionalized HBP 1 . The phase-separated HBPs assembled at the disclination lines; nevertheless, in the nematic phase, all of the defect lines oriented vertically with respect to the substrate surface in the thin cell. Thus, the phase-separated HBPs appeared to be dots under the microscope. For HBP $\mathbf{1 b}\left(M_{n} \sim 2.5 \times 10^{3}\right)$, phase separation also occurred, but the size of the dots was smaller in the chiral nematic phase in particular. However, for HBP 1c $\left(M_{n} \sim 1.6 \times 10^{3}\right)$, the HBPs did not aggregate at the disclination line in the chiral nematic phase (Figure 6f). Additionally, the characteristic brightness gradation of
Table 1 The molecular weights of the fractionalized HBPs

\begin{tabular}{lcccc}
\hline HBP & $M_{n}{ }^{a}$ & $M_{w}{ }^{a}$ & $M_{w} / M_{n}$ & $M_{n} / M_{\text {monomer }}$ \\
\hline 1 (unfractionalized) & $1.0 \times 10^{4}$ & $7.1 \times 10^{4}$ & 6.9 & 38 \\
1a & $4.4 \times 10^{3}$ & $5.2 \times 10^{3}$ & 1.2 & 17 \\
1b & $2.5 \times 10^{3}$ & $3.1 \times 10^{3}$ & 1.3 & 9.5 \\
1c & $1.6 \times 10^{3}$ & $2.9 \times 10^{3}$ & 1.8 & 6.0 \\
\hline
\end{tabular}

apolystyrene-reduced molecular weight.

fluorescence corresponding to the LC director variation in the nematic phase with Schlieren texture was observed in a confocal fluorescence polarizing micrograph (Figure 6c), which implies that the low-molecular-weight oligomers of $\mathrm{HBP} 1 \mathrm{c}$ were dissolved in $5 \mathrm{CB}$ not only in the isotropic phase but also in the nematic and chiral nematic phases. Moreover, the mesogenic perylenes linked to the HBPs were oriented along the LC directors.

For HBPs with high molecular weights, the particle size of an HBP molecule was much larger than the LC molecule, and the elastic energy of the LC molecules around the HBP molecules increased. Therefore, the HBPs were hardly soluble, and the molecules aggregated as micrometer-sized particles, as in the case of normal phase separation. However, for the HBPs with low molecular weights, the elastic energy of the LC molecules around the HBP molecules was assumed to be low because of the small size of the HBP molecules. The threshold value for dispersion in the oriented LC molecules was estimated to be $M_{n} \sim 2 \times 10^{3}$.

\section{CONCLUSIONS}

A novel synthesized perylene-labeled HBP with a mesogenic shell 1 was observed to migrate toward a field with a large distortion of directors. This polymer was captured by a disclination line of $1 / 2$ strength in nematic and chiral nematic phases. A well-aligned particle array of the HBPs was created using a chiral nematic phase confined in a wedge cell. Therefore, we have successfully controlled the spatial arrangement of HBP using the topological defect of liquid crystal directors. Because the orientation of liquid crystals can be varied by applying external fields, such as electric fields, magnetic fields, shear flow and temperature gradients, ${ }^{47,48}$ the arrangement of the particles induced by the director gradient could be varied by an external field, leading to an extensive expansion of the application fields of HBPs. 

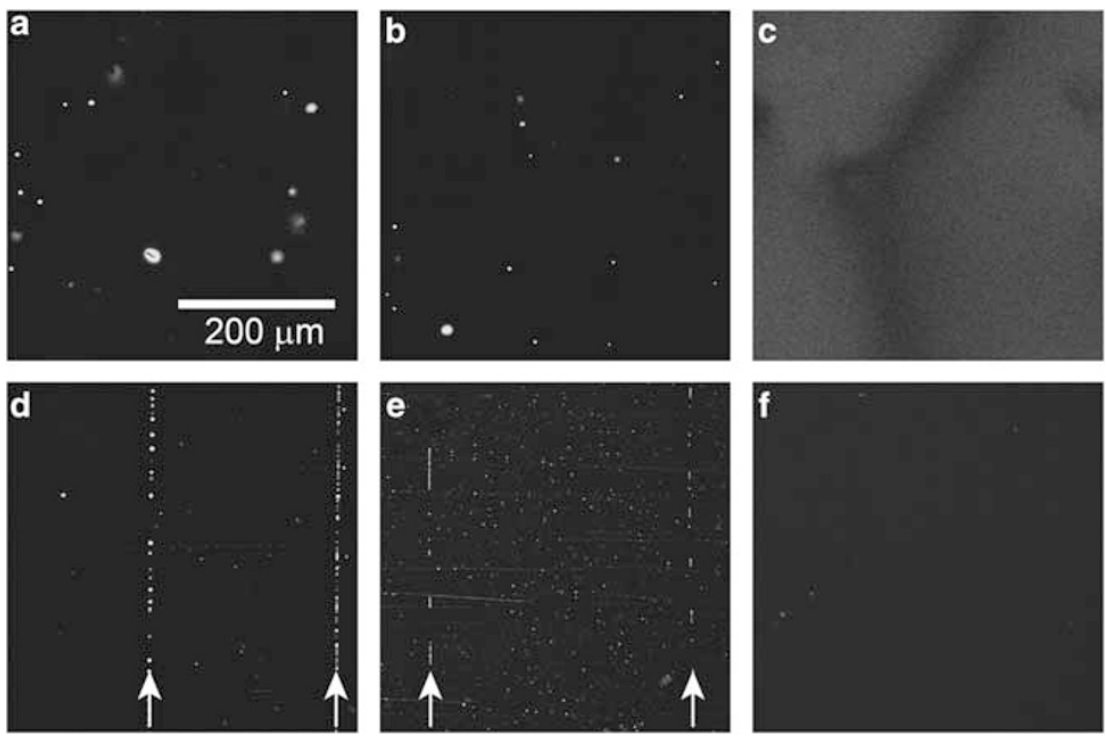

Figure 6 Fluorescence microscopic observations of HBP phase separation in the nematic and chiral nematic phases. (a) HBP 1a, (b) HBP 1b, and (c) HBP $\mathbf{1} \mathbf{c}$ in the nematic phase in thin glass cells. (d) HBP $\mathbf{1 a}$, (e) HBP $\mathbf{1 b}$, and (f) HBP $\mathbf{1 c}$ in the chiral nematic phase in wedge cells. The concentrations of the HBPs were changed appropriately to obtain clear images, and the chiral dopants used were different in each case.

\section{ACKNOWLEDGEMENTS}

This work was supported by Nissan Chemical Industries and a Grant-in-Aid for Scientific Research (No. 23107535) in Innovative Areas: 'Fusion Materials' (Area no. 2206) from MEXT. We also would like to thank Dr H Higuchi and Dr T Iwata for helpful discussions.

1 Hosono, H, Mishima, Y, Takezoe, H \& Mackenzie, K. J. D. Nanomaterials (Elsevier, Tokyo, 2006).

2 Ozin, G.A \& Arsenault, A.C Nanochemistry, A chemical approach to nanomaterials (RSC Publishing, Cambridge, 2005).

3 Voloschenko, D, Pishnnyak, O. P, Shiyanovskii, S. V \& Lavrentovich, O. D Effect of director distortions on morphologies of phase separation in liquid crystals. Phys. Rev. E. 65, 060701 (2002).

4 Loudet, J-C, Barois, P \& Poulin, P Colloidal ordering from phase separation in a liquidcrystalline continuous phase. Nature 407, 611-613 (2000).

5 Wang, S. W, Sprunt, S \& Chien, L. C Structure and morphology of polymer-stabilized cholesteric diffraction gratings. Appl. Phys. Lett. 76, 3516-3518 (2000).

6 Voloschenko, D \& Lavrentovich, O. D Light-induced director-controlled microassembly of dye molecules from a liquid crystal matrix. J. Appl. Phys. 86, 4843-4846 (1999).

7 Fleury, J-B, Pires, D \& Galerne, Y Self-connected 3D architecture of microwires. Phys. Rev. Lett. 103, 267801 (2009).

8 Škarabot, M, Ravnik, M, Žumer, S, Tkalec, U, Poberaj, I, Babič, D \& Muševič, I Hierarchical self-assembly of nematic colloidal superstructures. Phys. Rev. E. 77, 061706 (2008)

9 Škarabot, M \& Muševič, I Direct observation of interaction of nanoparticles in a nematic liquid crystal. Soft Matter 6, 5476-5481 (2010).

10 Muševič, I, Škarabot, M, Ravnik, M, Žumer, S, Poberaj, I \& Babič, D 2D interactions and binary crystals of dipolar and quadrupolar nematic colloids. Phys. Rev. Lett. 100, 217803 (2008).

11 Škarabot, M, Ravnik, M, Žumer, S, Tkalec, U, Poberaj, I, Babič, D, Osterman, N \& Muševič, I Interactions of quadrupolar nematic colloids. Phys. Rev. E. 77, 031705 (2008).

12 Pires, D, Fleury, J-B \& Galerne, Y Colloid particles in the interaction field of a disclination line in a nematic phase. Phys. Rev. Lett. 98, 247801 (2007).

13 Loudet, J. C, Hanusse, P \& Poulin, P Stokes drag on a sphere in a nematic liquid crystal. Science 306, 1525 (2004).

14 Koenig, Jr. G. M, Ong, R, Cortes, A. D, Antonio Moreno-Razo, J, de Pablo, J. J \& Abbott, N. L Single nanoparticle tracking reveals influence of chemical functionality of nanoparticles on local ordering of liquid crystals and nanoparticle diffusion coefficients. Nano Lett. 9, 2794-2801 (2009).

15 Stegmeyer, H Liquid Crystals (Steinkopff, Darmstadt, 1994).

16 Collings, P. J \& Hird, M Introduction to Liquid Crystals (Taylor \& Francis, London, 1997).

17 Ishizu, K Shibuya, T \& Mori, A Synthesis and characterization of hyperbranched poly(ethyl methacrylate) by quasi-living radical polymerization of photofunctional inimer. Polym. Int. 51, 424-428 (2002).
18 Ishizu, K \& Ohta, Y Architecture of nanocylinders with hyperbranched polymer side groups via living radical mechanism. J. Mater. Sci. Lett 22, 647-650 (2003).

19 Ishizu, K, Kojima, T, Ohta, Y \& Shibuya, T Nanopattern formation on polymer substrate using star-hyperbranched nanospheres. J. Colloid. Inter. Sci. 272, 76-81 (2004).

20 Ishizu, K, Park, J, Tanimura, K, Uchida, S \& Tamura, T Architecture of shell-crosslinked polymer nanoparticles via a living radical mechanism and modification to hollow nanocapsules. J. Mater. Sci. 39, 4353-4357 (2004).

21 Ishizu, K, Khan, R. A, Ohta, Y \& Furo, M Controlled radical polymerization of 2-hydroxyethyl methacrylate initiated by photofunctional 2-( $N, N$-diethyldithiocarbamyI)isobutyric acid. J. Polym. Sci.: Part A: Polym. Chem. 42, 76-82 (2004).

22 Ishizu, K, Shibuya, T, Park, J \& Uchida, S Novel synthesis and solution properties of hyperbranched poly(ethyl methacrylate)s by quasi-living radical copolymerization using photofunctional inimer. Polym. Int. 53, 259-265 (2004).

23 Ishizu, K \& Kakinuma, H Synthesis of nanocylinders consisting of graft block copolymers by the photo-induced ATRP technique. J. Polym. Sci.: Part A: Polym. Chem. 43, 63-70 (2005).

24 Craig, A. A \& Imrie, C. T Effect of spacer length on the thermal properties of side-chain liquid crystal polymethacrylates. 2 . synthesis and characterization of the poly[ $\omega$ - $(4$ 'cyanobiphenyl-4-yloxy)alkyl methacrylate]s. Macromolecules 28, 3617-3624 (1995).

25 Shibaev, V. P, Kostromin, S. G \& Plate, N. A Thermotropic liquid-crystalline polymers$\mathrm{VI}$ : comb-like liquid-crystalline polymers of the smectic and nematic types with cyanobiphenyl groups in the side-chains. Eur. Polym. J. 18, 651-659 (1982).

26 Han, X., Shanks, R. A. \& Pavel, D. Synthesis and characterisation of side-chain liquid crystalline poly[1-(\{[(4-cyano-4'-biphenyl)oxy]alkyl\}-oxy)-2,3-epoxypropane]. Macromol. Chem. Phys 205, 743-751 (2004).

27 Jánossy, I. Molecular interpretation of the absorption-induced optical reorientation of nematic liquid crystals. Phys. Rev. E. 49, 2957-2963 (1994).

28 Langhals, H., Karolin, J. \& Johansson, L.B.-A Spectroscopic properties of new and convenient standards for measuring fluorescence quantum yields. J. Chem. Soc. Faraday Trans. 94, 2919-2922 (1998).

29 Medvedeva, D., Bobrovski, A., Boiko, N., Shibaev, V., Shirinyan, V. \& Krayushkin, M. A Study of the photoorientation phenomena in cholesteric polymer systems containing photochromic diarylethene derivatives. Macromol. Chem. Phys. 207, 770-778 (2006).

30 Langhals, H. \& Saulich, S. Bichromophoric perylene derivatives: energy transfer from non-fluorescent chromophores. Chem. Eur. J. 8, 5630-5643 (2002).

31 Langhals, H. Control of the interactions in multichromophores: novel concepts. Perylene bisimides as components for larger functional units. Helv. Chim. Acta 88, 1309-1343 (2005).

32 Langhals, H. Chem. Ber. 118, 4641-4645 (1985).

33 Smalyukh, I. I., Shiyanovskii, S. V. \& Lavrentovich, O. D. Three-dimensional imaging of orientational order by fluorescence confocal polarizing microscopy. Chem. Phys. Lett. 336, 88-96 (2001).

34 Smalyukh, I. I. \& Lavrentovich, O. D. Three-dimensional director structures of defects in Grandjean-Cano wedges of cholesteric liquid crystals studied by fluorescence confocal polarizing microscopy. Phys. Rev. E. 66, 051703 (2002).

35 Ishizu, K. \& Mori, A. Synthesis of hyperbranched polymers by self-addition free radical vinyl polymerization of photo functional styrene. Macromol. Rapid Commun. 21, 665-668 (2000)

36 Wilson, T. Confocal Microscopy (Academic Press, London, 1990). 
37 Corle, T. R. \& Kino, G. S. Confocal Scanning Optical Microscopy and Related Imaging Systems (Academic Press, San Diego, CA, 1996).

38 Sheppard, C. J. R. \& Shotton, D. M. Confocal Laser Scanning Microscopy (BIOS Scientific Publishers, Oxford, 1997).

39 Pawley, J. B. Handbook of Biological Confocal Microscopy. 2nd edn (Plenum Press, New York, 1995)

40 Chestnut, M. H. Confocal microscopy of colloids. Curr. Opin. Colloid Interface Sci. 2, 158-161 (1997).

41 Tata, B. V. R. \& Raj, B. Confocal laser scanning microscopy: applications in material science and technology. Bull. Mater. Sci. 21, 263-278 (1998).

42 Ribbe, A. E. Laser scanning confocal microscopy in polymer science. Trends Polym. Sci. 5, 333-337 (1997).
43 Webb, R. H. Confocal optical microscopy. Rep. Prog. Phys. 59, 427-471 (1996). 44 Dierking, I. Fluorescence confocal polarizing microscopy: imaging liquid crystal director fields in three dimensions. ChemPhysChem 2, 663-664 (2001).

45 Held, G. A., Kosbar, L. L., Dierking, I., Lowe, A. C., Grinstein, G., Lee, V. \& Miller, R. D. Confocal microscopy study of texture transitions in a polymer stabilized cholesteric liquid crystal. Phys. Rev. Lett. 79, 3443-3446 (1997).

46 Malet, G. \& Martin, J. C. Optical study of the structure of grandjean lines in cholesterics with very large pitches. J. Phys. 40, 355-373 (1979).

47 de Gennes, P. G. \& Prost, J. The Physics of Liquid Crystals. 2nd edn (Clarendon, Oxford, 1993).

48 Demus, D., Goodby, J, Gray, G. W, Spiess, H.-W \& Vill, V. Handbook of Liquid Crystals (Wiley-VCH, Weinheim, 1998).

Supplementary Information accompanies the paper on Polymer Journal website (http://www.nature.com/pj) 